\title{
Nonlinear Effects on ENSO's Period
}

\author{
FIONA ECCLES \\ Atmospheric Oceanic and Planetary Physics, University of Oxford, Oxford, United Kingdom \\ ELI TZIPERMAN \\ Environmental Sciences, Weizmann Institute of Science, Rehovot, Israel
}

(Manuscript received 12 January 2003, in final form 19 August 2003)

\section{ABSTRACT}

\begin{abstract}
The dependence of ENSO's period on its amplitude is examined using a simple delayed oscillator model. This dependence is first calculated in the strongly nonlinear regime by extracting and analyzing unstable periodic orbits from the chaotic attractor of the model. In this regime, the period is found to decrease with increasing amplitude. Next, the dependence of the period on the amplitude is also calculated analytically and numerically in the weakly nonlinear regime by varying the ocean-atmosphere coupling coefficient. In this case, the period increases with the amplitude. The weakly nonlinear result reflects the dependence of the period on the oceanatmosphere coupling strength rather than the dependence on the amplitude, while the strongly nonlinear result is the robust take-home message here: the period reduces with increasing amplitude.
\end{abstract}

\section{Introduction}

Despite much progress in the understanding of El Niño-Southern Oscillation (ENSO), the dynamics that are responsible for its period, which seems to be quite robust at 3-5 yr, are as yet poorly understood. Proxy observations show a similar period even during the previous interglacial period, some $120 \mathrm{kyr}$ ago (Hughen et al. 1999), while other proxy evidence (Rodbell et al. 1999) indicates a possibly much longer period 700015000 years ago. The lack of understanding of what leads to ENSO's period is especially disconcerting given the failure of many state-of-the-art general circulation models (GCMs) to reproduce the correct period of ENSO (e.g., Timmermann et al. 1999). Most likely the beginning of such an understanding could come from a relatively simple model that can be understood in some detail.

The travel times of the first mode baroclinic equatorial Kelvin and Rossby waves involved in the delayed oscillator ENSO mechanism (Battisti 1988; Suarez and Schopf 1988) are in the range of 2-6 months, and time scales of mixed-layer processes are of the order of 2 months. These time scales are significantly shorter than the ENSO period itself, which therefore seems to be affected more by the ocean-atmosphere coupling strength than by the wave travel times.

Corresponding author address: Eli Tziperman, Department of Earth and Planetary Sciences and Division of Engineering and Applied Sciences, Harvard University, 20 Oxford St., Cambridge, MA 02138-2902. E-mail: eli@eps.harvard.edu
The issue of ENSO period has been discussed in numerous recent papers using models from GCMs to toy models. Codron et al. (2001) showed that ENSO period in a coupled GCM depends quite sensitively on the details of some model parameterizations. Similarly, Syu and Neelin (2000) used a hybrid coupled model to show that stronger vertical mixing leads to a longer ENSO period. Kirtman (1997) used an oceanic GCM (OGCM) coupled to a statistical atmosphere and showed that off equatorial Rossby waves are critical to getting the right period in this model. Proceeding to simpler models, Fedorov and Philander (2001) analyze the linear stability of the tropical Pacific similarly to Jin and Neelin (1993) and find different physical modes with different characteristic periods. Finally, Kang and An (1998) study the dependence of the period on the wave reflection coefficients in a simple equatorial model.

While it is not difficult to reproduce the 4-yr ENSO period in a simple linear delayed oscillator model by adjusting the ocean-atmosphere coupling coefficient, there are indications that nonlinear effects do play an important role. Münnich et al. (1991) suggested that nonlinear effects, more specifically a period-doubling bifurcation, lead to the 4-yr ENSO period, based on their simple delayed oscillator model. Nonlinear effects were also discussed by van der Vaart et al. (2000; see also Dijkstra 2000), who used numerical bifurcation methods to study a nonlinear coupled ocean-atmosphere set of equations, recognizing that one needs to deal simultaneously with the background state and the variability about this background. 
We do not know at the moment whether ENSO is self-sustained (hence nonlinear), possibly even chaotic (Jin et al. 1994; Tziperman et al. 1994, 1995), or perhaps it is a damped linear mode, excited by atmospheric forcing and efficiently amplified by nonnormal dynamics (Farrell 1989; Kleeman and Moore 1997; Penland and Sardeshmukh 1995). In this study we concentrate on the first possibility and examine nonlinear effects on ENSO's period. We use for this purpose the delayed oscillator ENSO model of Galanti and Tziperman (2000, hereafter GT) described in the next section.

More specifically the objective of this paper is to find out whether there is a relation between the period of ENSO and its amplitude, and what that relation is. That is, can we say that a series of stronger events would be characterized by a shorter period-or a longer period? The fact that such a simple question still does not have a simple answer clearly indicates that there is room for more work on the subject.

Now, we approach this question in two different parameter regimes and by using two different methods. First, we investigate the amplitude-period relation in a strongly nonlinear (in fact, chaotic) regime, using some subtle properties of a chaotic ENSO attractor. When the model is chaotic, it has many different perfectly periodic solutions that are, however, unstable and are known as "unstable periodic orbits" (UPOs). Each of these corresponds to an ENSO cycle with a different period and a different amplitude. We therefore look for UPOs in the chaotic attractor in order to deduce the desired amplitude-period relation. The motivation for this approach is as follows. In order to modify ENSO's amplitude and find what the relation to its period is, one could modify model parameters that affect the amplitude. However, changing these parameters may also have a direct effect on the ENSO period in the model, so we would not be able to isolate the effects on the period due to the amplitude change alone. In order to separate out the effect of the nonlinearity, we ran the model in a chaotic regime with one set of model parameters. By extracting and analyzing several UPOs, we can study the effect of the nonlinearity on ENSO's period for a single set of model parameters. It is important to note that the chaotic regime is merely a tool used here. The results regarding the effect of the nonlinearity on the period of ENSO may therefore also be valid if ENSO is in a nonchaotic regime.

Next, we examine the same issue in a weakly nonlinear parameter regime using an analytic derivation of a weakly nonlinear approximation. The results in the two different regimes are then compared and contrasted.

In section 2 we first briefly describe the model used here, and the method used to extract the model's unstable periodic orbits. Section 3 presents the deduced amplitude-period relationship in the delayed oscillator model found using the UPOs and attempts to explain these results in terms of the wave dynamics mechanism. Finally, we use a weakly nonlinear approximation to
TABle 1. Parameters in Eqs. (1) and (3).

\begin{tabular}{ll}
\hline \hline Parameter & \multicolumn{1}{c}{ Description } \\
\hline$h$ & Thermocline depth anomaly \\
$T$ & Temperature anomaly \\
$\varepsilon_{m}$ & Oceanic damping coefficient \\
$\tau_{K}$ & Kelvin wave basin crossing time \\
$\tau_{R}$ & Rossby wave basin crossing time \\
$r_{W}, r_{E}$ & Western/eastern reflection coefficients \\
$\tau_{\text {wind }}$ & Extent of travel time affected by wind stress \\
$\rho$ & Mean density of ocean \\
$A^{*}$ & Relating wind stress to SST \\
$b_{0}$ & Annual mean coupling strength \\
$\mu$ & Seasonal coupling coefficient \\
$w$ & Mean upwelling \\
$\varepsilon_{T}$ & Thermal damping coefficient \\
$T_{\text {sub }}$ & Temperature anomaly at $H_{1}$ \\
$h$ & Thermocline depth anomaly \\
\hline
\end{tabular}

derive an analytic amplitude-frequency relation for the same delayed oscillator model in section 4 , and conclude in section 5 .

\section{Model and methodology}

\section{a. The model}

The model we use in this study is that of GT. This is a delayed oscillator model derived from the recharge oscillator model of Jin (1997a,b) which in turn is derived from the model of Zebiak and Cane (1987, hereafter $\mathrm{CZ}$ ). The resulting delayed oscillator equation is, in fact, more similar to that originally derived by Suarez and Schopf (1988) than to the recharge oscillator. The equation for the thermocline depth anomaly in the eastern Pacific is

$$
\begin{aligned}
h(t)= & e^{-\varepsilon_{m}\left(\tau_{R}+\tau_{K}\right)} r_{W} r_{E} h\left(t-\tau_{R}-\tau_{K}\right) \\
& -e^{-\varepsilon_{m}\left(\tau_{K}+\tau_{R} / 2\right)} r_{W} \frac{1}{\beta \rho} A^{*} \tau_{\text {wind }} \tau_{R} \mu\left(t-\tau_{K}-\frac{\tau_{R}}{2}\right) \\
& \times b_{0} T\left(t-\tau_{K}-\frac{\tau_{R}}{2}\right) \\
& +\frac{1}{\rho C_{0}} \tau_{\text {wind }} \tau_{K} \mu\left(t-\frac{\tau_{K}}{2}\right) b_{0} e^{-\varepsilon_{m} \tau_{K} / 2} T\left(t-\frac{\tau_{K}}{2}\right),
\end{aligned}
$$

where the model parameters are described in Table 1; $\tau_{R}$ and $\tau_{K}$ are the basin crossing times for the Rossby and Kelvin waves, respectively; and $r_{W}, r_{E}$ are the reflection coefficients at the western and eastern boundaries; and $\beta=d f / d y$ is the gradient of the Coriolis force. The first term on the rhs of (1) corresponds to a "free" wave that left the eastern basin (after a reflection by the eastern boundary) at a time $t-\tau_{R}-\tau_{K}$ and traveled as a Rossby wave to the western boundary, was reflected and traveled back to the eastern boundary as a Kelvin wave, arriving to the eastern boundary at time $t$. The 

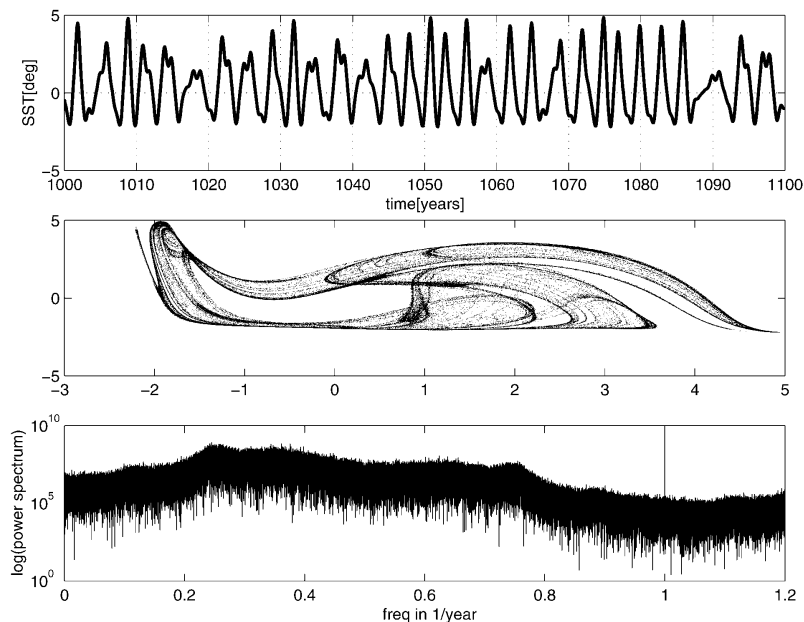

FIG. 1. (top) Time series of model temperature. (middle) The reconstructed delayed coordinate phase space $T(t)$ vs $T(t-\tau)$ where $\tau$ is $1 \mathrm{yr}$, and where $T(t)$ is also subsampled every year (Tziperman et al. 1995). (bottom) The corresponding frequency spectrum. Parameters for this plot are $b_{0}=7.5 \times 10^{10} \mathrm{~kg}$ month ${ }^{-2} \mathrm{~m}^{-1}{ }^{\circ} \mathrm{C}^{-1}$ and $\delta=0.18$.

second term represents a Rossby wave excited at a time $t-\tau_{K}-\tau_{R} / 2$ in the central Pacific, which traveled westward, was reflected, and traveled back as a Kelvin wave arriving again to the eastern Pacific at time $t$. The final term is a Kelvin wave, excited at a time $t-\tau_{K} / 2$ in the center of the basin and then traveled to the eastern basin. The ocean-atmosphere seasonal coupling is

$$
\mu=1+\delta \cos \left(w_{a} t-\varphi\right)
$$

$w_{a}=2 \pi / 12$ is the annual frequency, and $\varphi=5 \pi / 6$ is the phase; the coupling peaks in May due to factors such as the seasonal north-south movement of the intertropical convergence zone (ITCZ; Tziperman et al. 1997b).

The equation for the evolution of eastern Pacific SST is a simplification of the one from the $\mathrm{CZ}$ model and includes vertical advection and damping:

$$
\partial_{t} T=-\varepsilon_{T} T-\gamma \frac{\bar{w}}{H_{1}}\left[T-T_{\text {sub }}(h)\right],
$$

where $T_{\text {sub }}$ is the temperature anomaly at depth $H_{1}$ (the mean thermocline depth) and is approximated as a tanh function (Münnich et al. 1991).

Note that the main nonlinearity in the model is due to $T_{\text {sub }}$, consistent with the analysis of fuller models (Battisti 1988), and note also that this model is in what Jin and Neelin (1993) term the "mixed mode," including both the delay times due to the wave travel times and the time required for the SST to adjust to changes in $h$.

As explained above, we need to run the model in a chaotic regime in order to separate the role of nonlinearity from that of the parameters in setting the period of El Niño. This will become clearer as we proceed

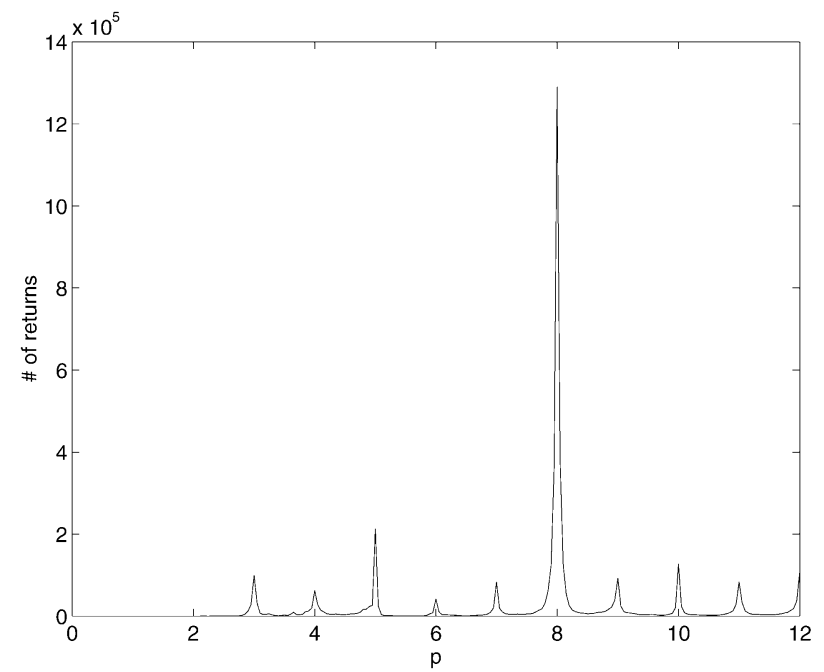

FIG. 2. Number of returns for each value of $p, \varepsilon=0.02$.

below. The GT model undergoes the quasi-periodic route to chaos, just like the fuller CZ model (Tziperman et al. 1994, 1995). The model solution in the chaotic regime is shown in Fig. 1.

\section{b. Finding unstable periodic orbits}

We would like to examine and compare several periodic solutions of our model equations that have different periods and amplitudes. When the model is in the chaotic regime, different periodic solutions exist as unstable periodic orbits embedded within the chaotic attractor seen in the middle panel of Fig. 1. Extracting and analyzing these solutions provides us with the desired different periodic solutions (i.e., ENSO cycles) for the same values of the model parameters.

To extract the UPOs, we ran the model in the chaotic regime for $100000 \mathrm{yr}$, with output every day to obtain a time series of the SST, $T(t)$, and then determined the UPOs in a three-dimensional delay-coordinate phase space reconstruction from the $T(t)$ time series following Tziperman et al. (1997a). The delay coordinates are defined as $\mathbf{X}(t)=\left\{X_{1}, X_{2}, X_{3}\right\}=\{T(t-2 \tau), T(t-\tau)$, $T(t)\}$ where $\tau$ is 1 yr. For a given period $p$, we search for phase space points $\mathbf{X}(t)$ that retuned to the same neighborhood after a period $p$, so that $\| \mathbf{X}(t)-\mathbf{X}(t-$ $p) \|<\varepsilon$ for some small $\varepsilon$. The phase space points that satisfy this condition are referred to as near-return points. When plotting the number of phase space points that satisfy this criterion against $p$, the UPOs show up as peaks (Fig. 2). The points $\mathbf{X}(t)$ that satisfy the above criterion for $p=3,4,5$ and $6 \mathrm{yr}$ are shown in Fig. 3.

A close examination of Fig. 3 shows that there are two separate 3-yr UPOs (the two loops seen in Fig. 3 are independent and do not cross). There are also two 6-yr UPOs. Each of them is composed of a double loop that is very similar to the $3-\mathrm{yr}$ UPO. Thus the 6-yr UPO is nothing but a result of a period doubling bifurcation 
(a)

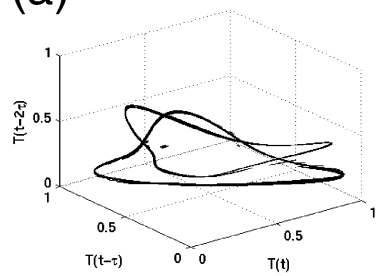

(c)

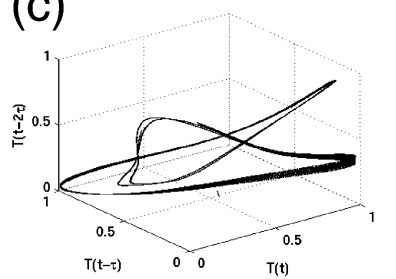

(b)

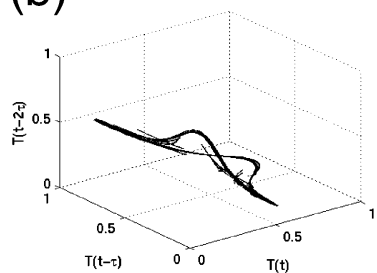

(d)

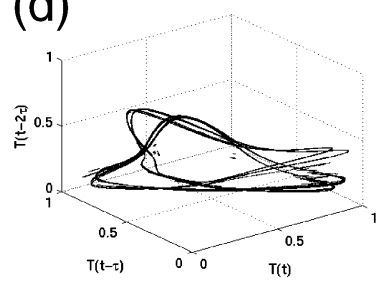

FIG. 3. Reconstructed phase space points that lie on UPOs for period of (a) $p=3$, (b) $p=4$, (c) $p=5$, and (d) $p=6 \mathrm{yr}$.

of the 3-yr UPO. Given the near-return points composing each of the different UPOs, we can extract a continuous portion of the time series $\mathbf{X}(t)$ that is composed of near returns, and that therefore lies very near a given UPO. This allows us to also examine the corresponding time series for the temperature and thermocline depth, as well as examine the different terms in the model equations along the UPOs, as will be seen below. Subsequently, these continuous time series segments will allow us to also study the wave dynamics for each of the UPOs. The chosen time series segments for $\mathbf{X}(t)$ are shown in Fig. 4, with the corresponding temperature time series in Fig. 5.

Our objective requires that we examine the period versus amplitude of the different UPOs. Now some of the UPOs extracted from the chaotic model attracter represent more than a single ENSO cycle and are the results of the merging of two or more UPOs. For example, Figs. 4 and 5 make it clear that the 5 -yr orbit is composed of a 2-yr orbit and a 3-yr orbit joined together. Thus, the 5-yr periodic solution reflects two ENSO cycles, one with a 2-yr period, and one with a 3-yr cycle. These 2- and 3-yr cycles are not exactly periodic, of course, as they are joined to form a periodic 5-yr cycle. Similarly, each of the 6-yr UPOs is made of two 3-yr UPOs, and the 8-yr UPO is also composed of shorter period cycles. In order to construct the amplitude-period relation for the different UPOs, it would clearly not be useful to treat the merged 5-yr period, for example, as if it were an independent 5-yr period UPO. We can only use those UPOs that correspond to a single ENSO cycle, which leaves the 3- and 4-yr UPOs on which we concentrate next.

The chaotic motion of the model corresponds to irregular jumps between the different UPOs [not just those extracted here; see Tziperman et al. (1994)]. With no seasonal cycle present, the model is periodic rather than

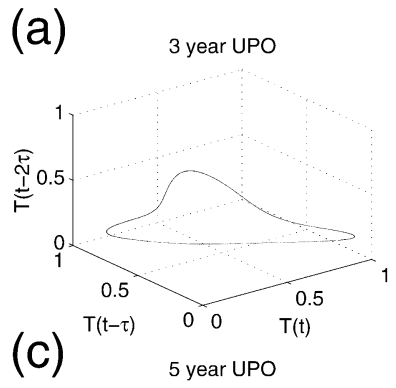

(b) 4 year UPO
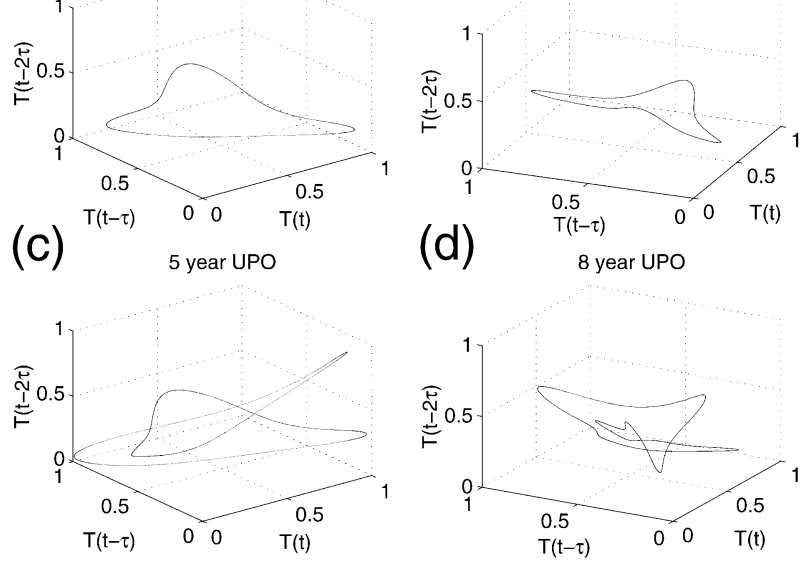

FIg. 4. Time series for segments of $\mathbf{X}(t)$ that lie near the UPOs for (a) 3, (b) 4, (c) 5, and (d) 8 yr.

chaotic, and the ENSO period is about $4 \mathrm{yr}$. It is no surprise therefore that once seasonal forcing is added and the system is chaotic, it tends to spend most of its time in the attractor near the 3-, 4-, and 5-yr UPOs, which are therefore also the least unstable ones.

\section{An amplitude-period relation in the strongly nonlinear regime}

Figure 6 directly compares the 3- and 4-yr UPOs and clearly suggests that the larger the ENSO amplitude, the

(a)

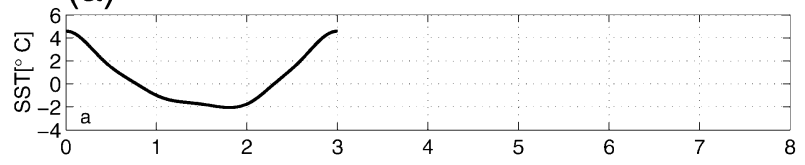

(b)

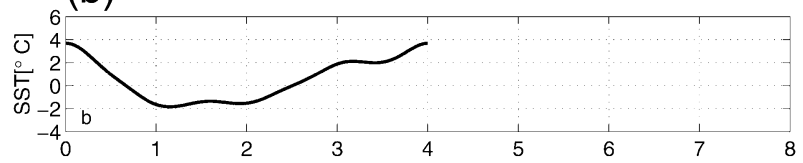

(c)

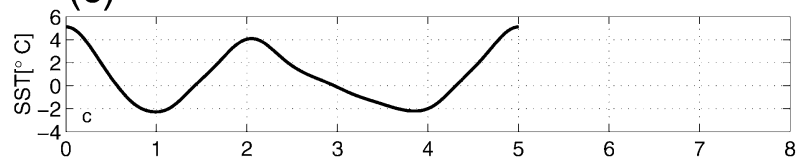

(d)

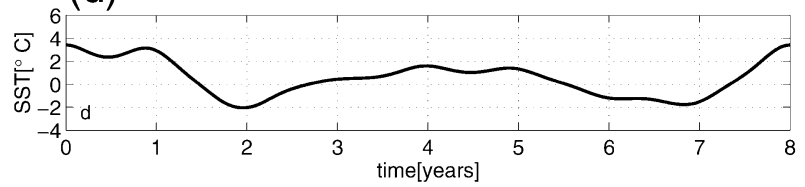

FIG. 5. Time series of SST for the same solutions seen in Fig. 4 for the UPOs of (a) 3, (b) 4, (c) 5, and (d) 8 yr. 

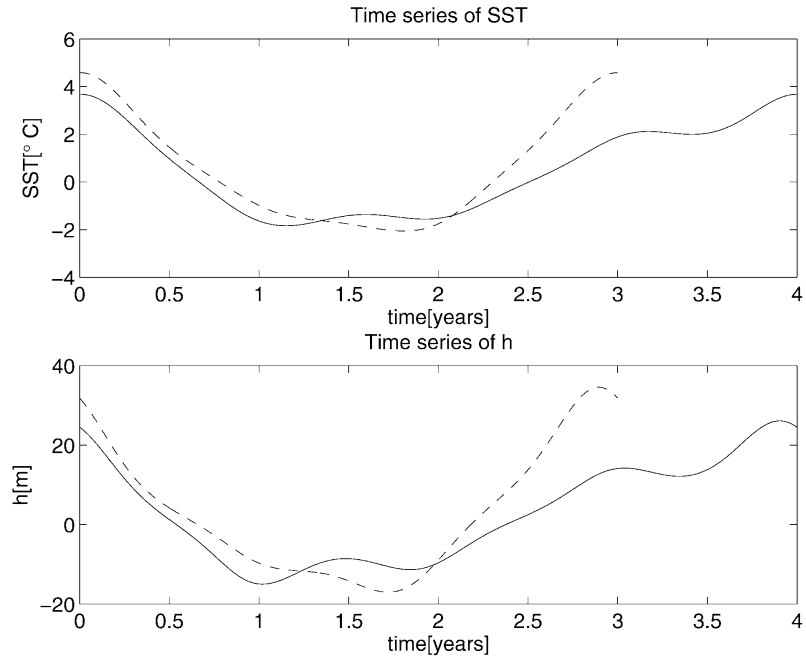

FIG. 6. SST and $h$ for the 3-yr (dashed) and 4-yr (solid) UPOs.

shorter is its period. This seems to be the case also for the 2-yr cycle (not UPO) that is part of the 5-yr UPO and is seen in Fig. 5c. This 2-yr cycle has a yet larger amplitude than either the 3- or 4-yr UPOs. These results indicate that the larger is ENSO's amplitude (in this model), the shorter is its period. We next try to rationalize this in terms of a wave dynamics mechanism.

Figure 6 shows that the 4-yr cycle spends time between 1 and $2 \mathrm{yr}$ at an approximately constant temperature, and the warming phase of the 4-yr UPO, which begins around $2 \mathrm{yr}$, is longer and more gradual. To attempt to explain this behavior in terms of wave dynamics we examined the various terms in (1), and they are plotted for each UPO in Fig. 7. We use the following notation for the terms from (1) for the thermocline depth anomaly. The free Rossby-Kelvin wave is denoted by RK, ER is the excited Rossby wave, and EK is the excited Kelvin wave [see discussion after (1)].

Given that $\tau_{R}$ is 8.5 months and $\tau_{K}$ is 2.1 months (GT), the effect on $h$ at any point in time is determined by the SST 1 month previously (via term EK) and by the SST about 6 months previously (via term ER). In Fig. 7 it can be seen that the Rossby wave term always lags the Kelvin wave term by about 6 months (and its amplitude is smaller and with an opposite sign). Additionally, the free wave term RK depends on $h 10.6$ months previously. To complement the picture, Fig. 8 shows the terms in the temperature equation (3) for the 3 - and 4-yr UPOs. In these figures, the different plotted terms correspond to

$$
\begin{aligned}
\text { term } 1 & =-\varepsilon_{T} T, \\
\text { term } 2 & =-\gamma \frac{\bar{w}}{H_{1}} T, \\
\text { term } 3 & =\gamma \frac{\bar{w}}{H_{1}} T_{\text {sub }}(h) .
\end{aligned}
$$
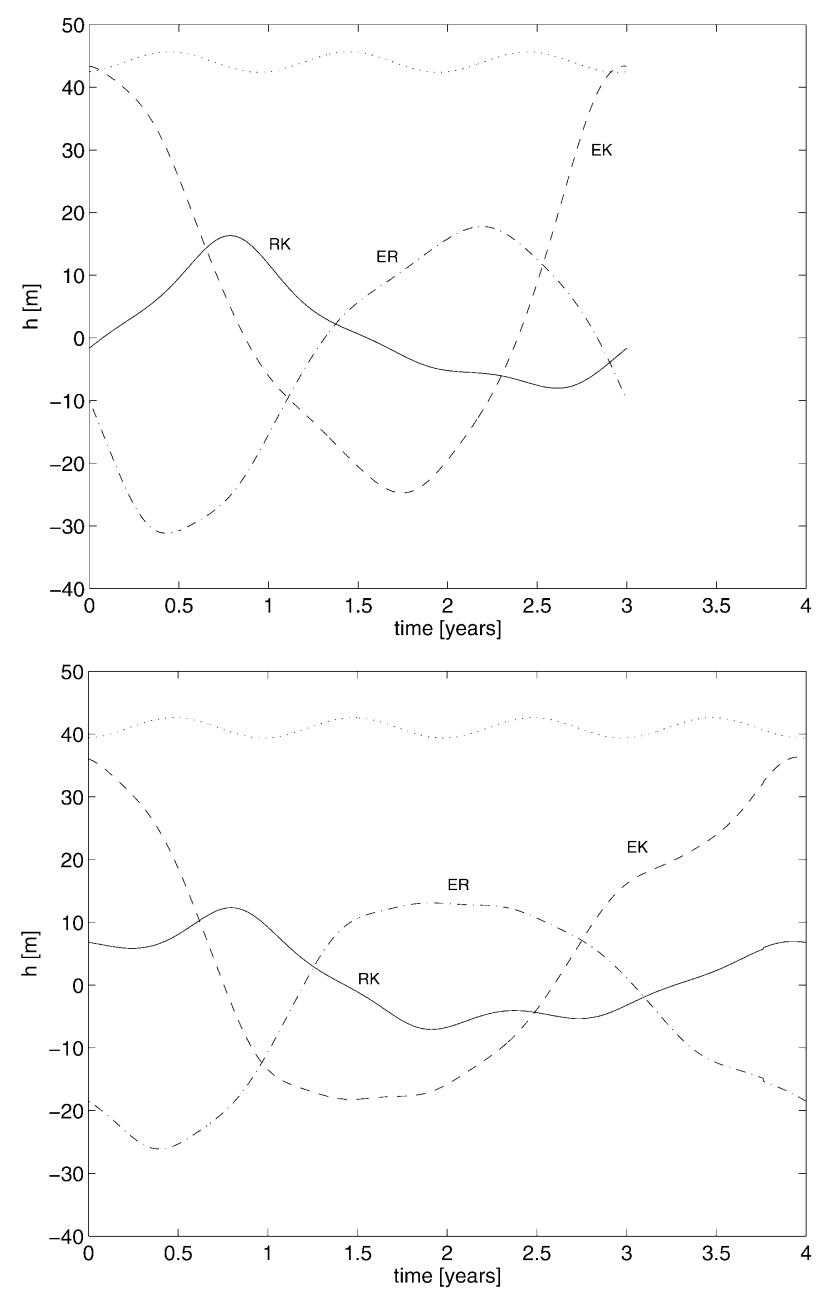

FIG. 7. Terms in Eq. (1) for the thermocline depth anomaly, for the (top) 3-yr UPO and (bottom) 4-yr UPO: RK(-) ER(--), EK(--). The sine wave time series shows the coupling coefficient from Eq. (2) during the UPO.

The 3-yr cycle and the 4-yr cycle seem to develop quite similarly for the first $2 \mathrm{yr}$ (Fig. 6). At that point, however, the temperature and thermocline depth anomaly in the 3-yr cycle proceed to increase rapidly, while those in the 4-yr cycle increase more gradually and take one more year to complete the cycle. The behavior of the different terms in the thermocline depth equation depends on the temperature and depth at earlier times via the various delay terms. The key to the different behaviors past the first $2 \mathrm{yr}$ is therefore what happens during the second year in both cycles.

Note that the 4-yr cycle reaches its La Niña maximum at about year 1, earlier than the 3-yr cycle, which for which La Niña peaks just before year 2. Right after the La Niña peak, the temperature and thermocline depth of the 4-yr cycle start increasing, but then stall. Because the 4-yr cycle stalls toward the end of the second year, it has a smaller negative amplitude than the 3 -yr cycle at that point. This weakens the feedback loop for the 4- 

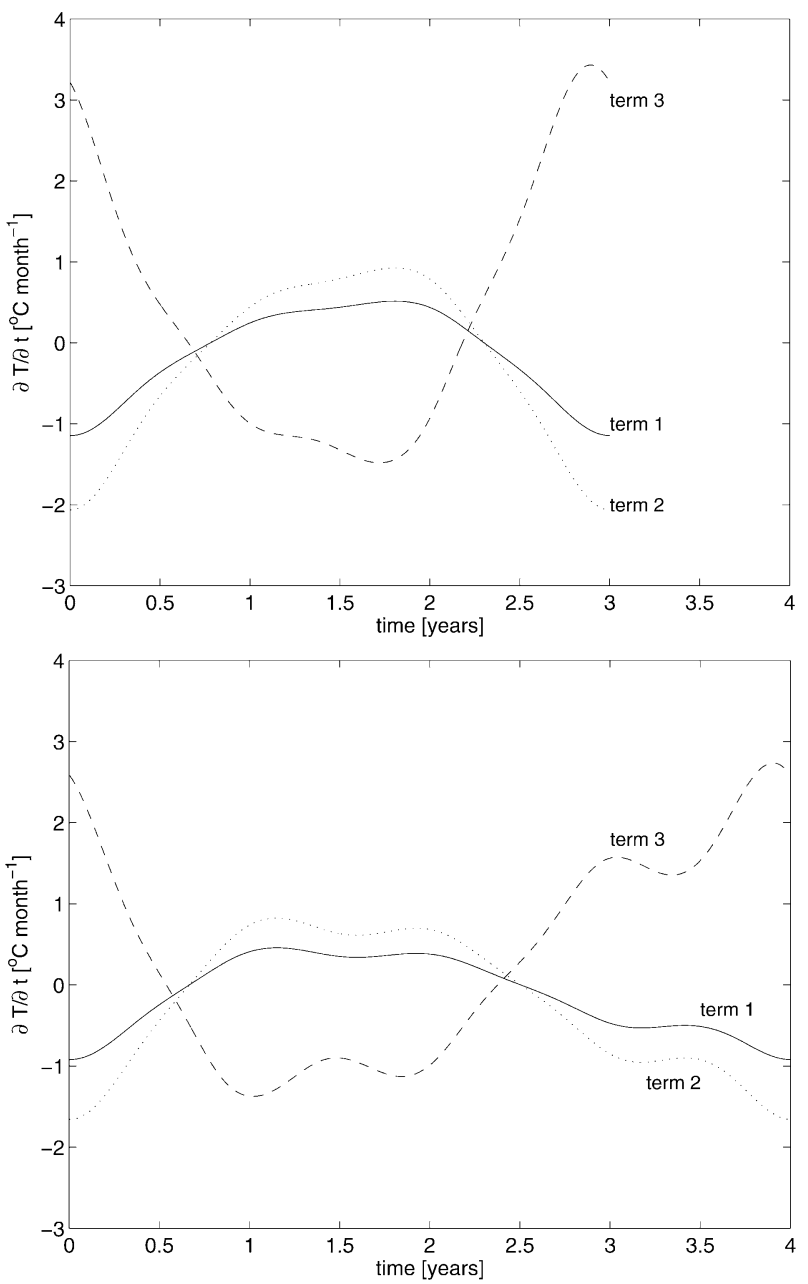

FIG. 8. Terms in Eq. (1) for the SST anomaly, for the (top) 3-yr UPO and (bottom) 4-yr UPO.

yr cycle during the coming months, as can be seen by the fact that the Kelvin wave term is larger and grows more rapidly during the third year in the $3-y r$ cycle than in the 4-yr cycle (Fig. 7). The 4-yr cycle therefore gets slower into the El Niño phase and takes one more year to complete.

The key to the slower development of the 4-yr cycle is therefore the halt of the cooling of the Kelvin wave at the end of the first year. The 4-yr event being weaker is clearly consistent with the fact that the Kelvin and Rossby waves are weaker. This weakness makes the event develop more slowly, and in particular shift from phase to phase more slowly, as described above. The stronger 3-yr event sustains stronger Kelvin and Rossby terms, which are able to shift the system from El Niño to La Niña and back to El Niño faster. This wave dynamics perspective gives us some intuition regarding the physical processes responsible for the deduced amplitude-period relation for the delayed oscillator model used here.

\section{An amplitude-period relation in the weakly nonlinear limit}

The previous section presented the amplitude-period relation obtained in the strongly nonlinear case. We wish to complement this with a different parameter regime in which the nonlinearity is weak and the dynamics nearly linear. Of course, in the purely linear case, one expects the period not to depend on the amplitude. But assuming that nonlinearity is small, allows us to make some progress analytically. A similar analysis using a different approach from ours was carried out in the appendix of Jin (1997a), where a multiple-scale analysis was used to deduce an amplitude-period relation in a weakly nonlinear regime. We shall return to Jin's results shortly.

Start from the delayed oscillator equations of GT, neglect seasonality in the coupling coefficient and expand the main nonlinearity [due to the hyperbolic tangent structure of $T_{\text {sub }}(h)$ ] keeping only the first nonlinear correction:

$$
\begin{aligned}
h(t) & =a h\left(t-\tau_{a}\right)+\mu b T\left(t-\tau_{b}\right)+\mu c T\left(t-\tau_{c}\right), \\
\frac{\partial T}{\partial t} & =-\varepsilon_{T} T-\frac{\bar{w}}{H_{1}} \gamma\left[T-T_{\text {sub }}(h)\right] \\
& \approx-p T+r h-s h^{3},
\end{aligned}
$$

where $\mu$ is an order- 1 coupling coefficient.

Substitute a solution that is of the most general form for the linear part of the problem,

$$
\left(\begin{array}{l}
T \\
h
\end{array}\right)=\left[\begin{array}{c}
A \cos (\omega t) \\
B \cos (\omega t+\phi)
\end{array}\right] e^{\lambda t},
$$

and assume that the model is neutrally stable $(\lambda=0)$, which implies some special relation between the model coefficients $a, b, c, \mu, p, r, s, \tau_{a}, \tau_{b}, \tau_{c}$. Next, substitute (6) in (4) and (5), multiply each of the resulting equations first by $\sin (\omega t)$ and then by $\cos (\omega t)$, and integrate over a full period $\int_{0}^{2 \pi / \omega} d t$. Furthermore, use

$$
\cos ^{3}(\theta)=\frac{3}{4} \cos (\theta)+\frac{1}{4} \cos (3 \theta)
$$

and neglect the $\cos (3 \omega t)$ term, equivalent to projecting our solution on the first Fourier term, and consistent with the fact that we did not include a $\cos (3 \omega t)$ term in (6). The above procedure results in the four equations:

$$
\begin{aligned}
0= & -p A+r B \cos \phi-\frac{3}{4} s B^{3} \cos \phi, \\
\omega A= & r B \sin \phi-\frac{3}{4} s B^{3} \sin \phi, \\
B \cos \phi= & a B \cos \left(\phi-\omega \tau_{a}\right)+\mu b A \cos \left(\omega \tau_{b}\right) \\
& +\mu c A \cos \left(\omega \tau_{c}\right), \\
B \sin \phi= & a B \sin \left(\phi-\omega \tau_{a}\right)-\mu b A \sin \left(\omega \tau_{b}\right) \\
& -\mu c A \sin \left(\omega \tau_{c}\right) .
\end{aligned}
$$



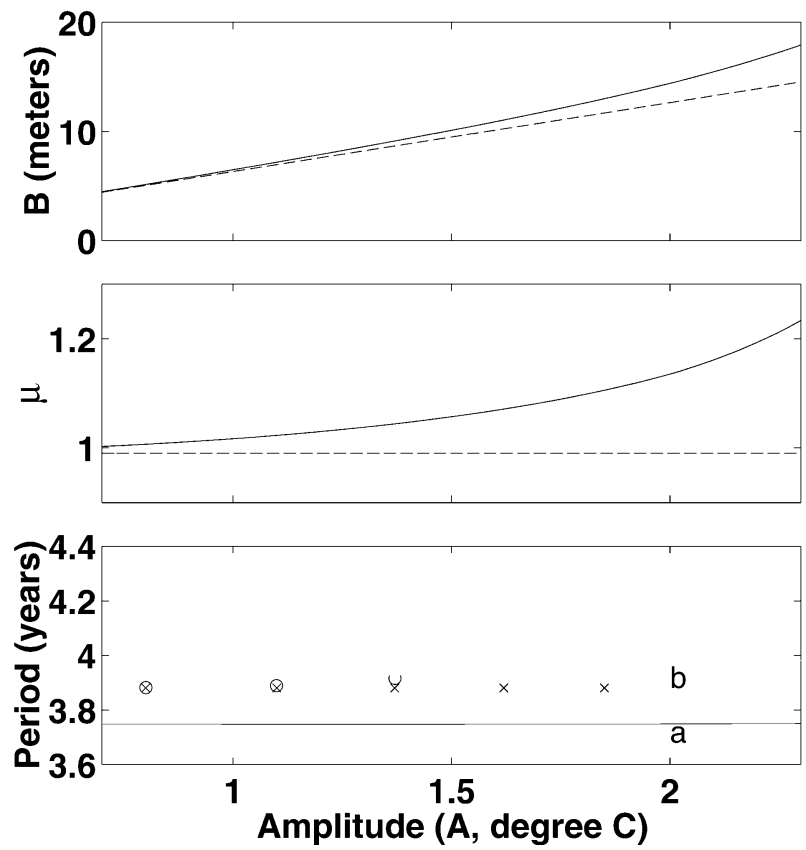

FIG. 9. Solution of the weakly nonlinear approximation [Eqs. (8)(11)] as a function of the SST anomaly amplitude $A$. (top) Amplitude of thermocline depth anomaly $B$ for the linear $(s=0$, dashed) and nonlinear ( $s \neq 0$, solid) cases. (middle) The coupling coefficient $\mu$ for the linear and nonlinear cases. (bottom) The period as function of amplitude $A$ for the linear (curve a) and nonlinear (curve b) cases. Also shown are results of numerical solution of the model equations $(4)$ and $(5)$ in the linear $(X)$ and nonlinear $(O)$ cases.

We would like to use these equations to find out what is the correction to the frequency due to the small nonlinear, cubic in $B$, term in the SST equation (5). This is essentially a weakly nonlinear correction to the linear analysis of Suarez and Schopf (1988), who used a somewhat simpler delayed oscillator model. For this purpose, fix the overall amplitude $A$ and solve this system for the unknown $B, \omega_{0}, \phi_{0}$, and $\mu$. The solution is obtained numerically using an optimization routine in MATLAB.

The upper panel of Fig. 9 shows the amplitude of the thermocline depth $B$ as a function of the amplitude of the SST anomaly $A$ for both the case where the nonlinearity is ignored $(s=0)$ and when it is included. In the linear case (dashed line), $B$ varies linearly with $A$, as expected. In the nonlinear case (solid line), the nonlinear effects start to play a significant role only for $A>1{ }^{\circ} \mathrm{C}$ or so. The lower panel shows the period as function of the amplitude $A$, again for both the linear (curve a) and nonlinear (curve b) cases. For a model amplitude above $0.6^{\circ} \mathrm{C}$, for which nonlinear effects are starting to be more significant, both the linear and nonlinear equations show a smooth dependence of the period on the amplitude. One can see that the period does not vary with the amplitude for the linearized model (Fig. 9, curve a), but increases with the amplitude when the nonlinear term is included in the weakly nonlinear analysis (curve b).
We now compare these results of the weakly nonlinear approximation to a numerical solution of (4) and (5). For this purpose, we first set the nonlinear term zero, $s$ $=0$, and also set the model coupling parameter $\mu$ such that the model is oscillating at a constant amplitude, in a neutrally stable regime. When the nonlinear terms in the delayed oscillator equation is set to zero, the amplitude of the oscillation depends on the initial perturbation used to initialize the model. This dependence is used to vary the amplitude of the oscillations in this linearized case, so the coupling coefficient remains constant in the linear case as the amplitude varies. As seen by the $\times$ marks in the lower panel of Fig. 9, the period is found not to depend on the amplitude in this linear case, just as in the weakly nonlinear approximation used above.

Next, we set $s \neq 0$ run the model for a few different values of the coupling coefficient $\mu$, and calculate the period and amplitude from the numerical solution. The results are shown by the circles in Fig. 9. The numerical results agree with the weakly nonlinear approximation: the model period increases with the amplitude when the nonlinearity is included.

Note that the weakly nonlinear approximation slightly underestimates the effects of the nonlinearity on the period, as the distance between curves a and b (of the approximation results) seems to grow less rapidly with the amplitude than the distance between the crosses and the circles (plotted from the numerical results). Overall, apart from a relatively small constant shift in the period, the weakly nonlinear approximation does quite well. For larger yet coupling coefficient and amplitude, where the weakly nonlinear approximation is no longer valid, the numerical solution indicates that the equations undergo another bifurcation and the period increases significantly.

\section{Comparison to strongly nonlinear results}

Clearly the results found in this section for the weakly nonlinear regime, both approximate and numerical, are opposite to those found earlier, in section 3, for the strongly nonlinear regime. In the latter regime, based on the analysis of the UPOs, we found that the period decreased with the amplitude, while in the weakly nonlinear case, the period is increasing with the amplitude. In order to explain this difference, we refer the reader to the appendix of Jin (1997a), in which a different method of analysis is applied to the weakly nonlinear regime. Jin finds there that the period indeed increases with increasing coupling coefficient (and amplitude), as we do here. By performing this analysis for both the linear and nonlinear cases (his Fig. A1), he also shows that the nonlinearity itself tends to shorten the period, as we find in our strongly nonlinear case here. We may therefore conclude that the response of the period to increasing amplitude, which we see here in the weakly nonlinear regime is, in fact, a response to the changing 
coupling coefficient, which has to be modified as part of the weakly nonlinear analysis. This leaves us with the results of the strongly nonlinear case as the more reliable and robust take-home message of this paper: the period tends to decrease with increasing amplitude.

\section{Conclusions}

The mechanisms leading to ENSO's robust 4-yr period (indicated by the observed broad spectral maximum at this period) are not yet fully understood, and GCMs tend to fail simulating the correct ENSO period. It is therefore important to explore issues related to ENSO's period, what determines it, and how it might vary with various climate parameters, using a full hierarchy of models. We have examined the relation between the period of ENSO and its amplitude in a simple delayed oscillator model. This was done first in a strongly nonlinear, chaotic regime, and then in a weakly nonlinear regime. In the strongly nonlinear regime, we found that the larger the amplitude of the ENSO cycle, the shorter its period. In contrast, we found that for the weakly nonlinear regime, the period increases with the amplitude.

The strongly nonlinear regime explored here would be relevant to the actual ENSO dynamics should it turn out that ENSO is self-sustained, and it would certainly be even more relevant if it turns out that ENSO's irregularity is due to low-order chaos. The weakly nonlinear regime explored here would be relevant if ENSO is largely linear and if nonnormal amplification of stochastic forcing is indeed the source of ENSO's existence and irregularity. In this later case the nonlinearity enters mostly when the events are near their peak and the dynamics are linear at other times.

Now, we obtained different results for the weakly and strongly nonlinear regimes in terms of the response of the period to the amplitude. We also explain in section 4 that the weakly nonlinear results are due to the variation of the ocean-atmosphere coupling coefficient, which is part of this analysis. Thanks to the use of UPOs in the chaotic attractor of the model, the strongly nonlinear analysis presented here does not require changing any model parameters as part of the examination of the amplitude-period relation. We therefore conclude that the results of the strongly nonlinear case are the takehome message of this paper: the period tends to decrease with increasing amplitude. It is important to emphasize that it is not the larger amplitude that causes the shorter period, nor vice versa. The dynamics have to adjust both the period and the amplitude to be consistent with each other according to the amplitude-period relation found here.

The short instrumental record makes it difficult to decide how important the nonlinear effects are to ENSO's dynamics. It would therefore be interesting to examine the amplitude-period relation in models of higher complexity, such as intermediate models or full GCMs, and perhaps eventually with the data as well.

Acknowledgments. We thank Fei-Fei Jin for his most illuminating comments, that helped us clarify the link between the weakly and strongly nonlinear results. We also thank two anonymous reviewers for their constructive comments. We thank Eli Galanti for his code and help, and Joe Keller for proposing the use of the weakly nonlinear approximation. Thanks also to Bill Young and Lenny Smith for their suggestions. ET was partially supported for this work by the Israeli Science Foundation.

\section{REFERENCES}

Battisti, D. S., 1988: Dynamics and thermodynamics of a warming event in a coupled tropical atmosphere-ocean model. J. Atmos. Sci., 45, 2889-2919.

Codron, F., A. Vintzileos, and R. Sadourny, 2001: Influence of mean stage changes on the structure of ENSO in a tropical coupled GCM. J. Climate, 14, 730-742.

Dijkstra, H. A., 2000: Nonlinear Physical Oceanography. Kluwer Academic, $456 \mathrm{pp}$.

Farrell, B., 1989: Optimal excitation of baroclinic waves. J. Atmos. Sci., 46, 1193-1206.

Fedorov, A. V., and S. G. Philander, 2001: A stability analysis of tropical ocean-atmosphere interactions: Bridging measurements and theory for El Niño. J. Climate, 14, 3086-3101.

Galanti, E., and E. Tziperman, 2000: ENSO's phase locking to the seasonal cycle in the fast SST, fast wave, and mixed mode regimes. J. Atmos. Sci., 57, 2936-2950.

Hughen, K. A., D. P. Schrag, S. B. Jacobsen, and W. Hantoro, 1999: El Niño during the last interglacial period recorded by a fossil coral from Indonesia. Geophys. Res. Lett., 26, 3129-3132.

Jin, F.-F., 1997a: An equatorial ocean recharge paradigm for ENSO. Part I: Conceptual model. J. Atmos. Sci., 54, 811-829.

_ $1997 \mathrm{~b}$ : An equatorial ocean recharge paradigm for ENSO. Part II: A stripped-down coupled model. J. Atmos. Sci., 54, 830-847.

- and D. Neelin, 1993: Modes of interannual tropical oceanatmosphere interaction-A unified view. Part I: Numerical results. J. Atmos. Sci., 50, 3477-3503.

- - - and M. Ghil, 1994: ENSO on the devil's staircase. Science, 264, 70-72.

Kang, I. S., and S. I. An, 1998: Kelvin and Rossby wave contributions to the SST oscillation of ENSO. J. Climate, 11, 2461-2469.

Kirtman, B. P., 1997: Oceanic Rossby wave dynamics and the ENSO period in a coupled model. J. Climate, 10, 1690-1704.

Kleeman, R., and A. M. Moore, 1997: A theory for the limitation of ENSO predictability due to stochastic atmospheric transients. $J$. Atmos. Sci., 54, 753-767.

Münnich, M., M. A. Cane, and S. E. Zebiak, 1991: A study of selfexcited oscillations of the tropical ocean-atmosphere system. Part II: Nonlinear cases. J. Atmos. Sci., 48, 1238-1248.

Penland, C., and P. D. Sardeshmukh, 1995: The optimal-growth of tropical sea-surface temperature anomalies. J. Climate, 8, 19992024.

Rodbell, D. T., G. O. Seltzer, D. M. Anderson, M. B. Abbott, D. B. Enfield, and J. H. Newman, 1999: An approximately 15,000year record of El Niño-driven alluviation in southwestern Ecuador. Science, 283, 516-520.

Suarez, M. J., and P. S. Schopf, 1988: A delayed action oscillator for ENSO. J. Atmos. Sci., 45, 3283-3287.

Syu, H. H., and J. D. Neelin, 2000: ENSO in a hybrid coupled model. Part I: Sensitivity to physical parameterizations. Climate Dyn., 16, 19-34.

Timmermann, A., J. Oberhuber, A. Bacher, M. Esch, M. Latif, and 
E. Roeckner, 1999: Increased El Niño frequency in a climate model forced by future greenhouse warming. Nature, 398, 694697.

Tziperman, E., L. Stone, M. A. Cane, and H. Jarosh, 1994: El-Niño chaos: Overlapping of resonances between the seasonal cycle and the Pacific ocean-atmosphere oscillator. Science, 264, 7274.

- M. A. Cane, and S. E. Zebiak, 1995: Irregularity and locking to the seasonal cycle in an ENSO prediction model as explained by the quasi-periodicity route to chaos. J. Atmos. Sci., 52, 293306.
_ - H. Scher, S. E. Zebiak, and M. A. Cane, 1997a: Controlling spatiotemporal chaos in a realistic El Niño prediction model. Phys. Rev. Lett., 79, 1034-1037.

_, S. E. Zebiak, and M. A. Cane, 1997b: Mechanisms of seasonalENSO interaction. J. Atmos. Sci., 54, 61-71.

van der Vaart, P. C. F., H. A. Dijkstra, and F. F. Jin, 2000: The Pacific cold tongue and the ENSO mode: A unified theory within the Zebiak-Cane model. J. Atmos. Sci., 57, 967-988.

Zebiak, S. E., and M. A. Cane, 1987: A model El Niño-Southern Oscillation. Mon. Wea. Rev., 115, 2262-2278. 